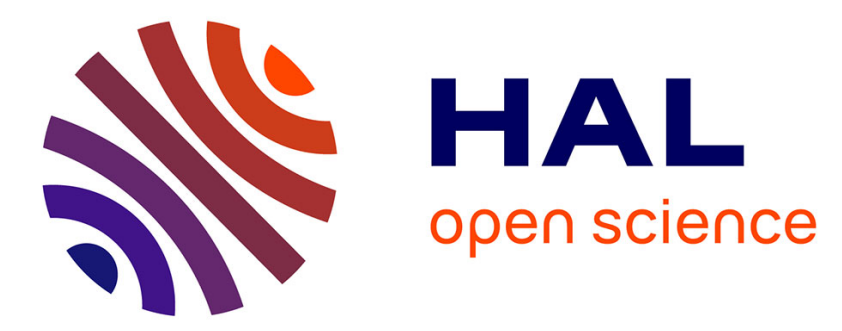

\title{
A flat-topped leaky-wave source for phased arrays with reduced scan losses
}

Francesco Scattone, Mauro Ettorre, Ronan Sauleau, N. J. G. Fonseca

\section{To cite this version:}

Francesco Scattone, Mauro Ettorre, Ronan Sauleau, N. J. G. Fonseca. A flat-topped leaky-wave source for phased arrays with reduced scan losses. EuCAP 2014, Apr 2014, The Hague, Netherlands. pp.1220 - 1224 10.1109/EuCAP.2014.6901995 . hal-01114112

\section{HAL Id: hal-01114112 \\ https://hal.science/hal-01114112}

Submitted on 18 Dec 2015

HAL is a multi-disciplinary open access archive for the deposit and dissemination of scientific research documents, whether they are published or not. The documents may come from teaching and research institutions in France or abroad, or from public or private research centers.
L'archive ouverte pluridisciplinaire HAL, est destinée au dépôt et à la diffusion de documents scientifiques de niveau recherche, publiés ou non, émanant des établissements d'enseignement et de recherche français ou étrangers, des laboratoires publics ou privés. 


\title{
A Flat-Topped Leaky-Wave Source for Phased Arrays with Reduced Scan Losses
}

\author{
F. Scattone ${ }^{1}$, M. Ettorre ${ }^{1}$, R. Sauleau ${ }^{1}$, N. J. G. Fonseca ${ }^{2}$ \\ ${ }^{1}$ Institut d'Electronique et de Télécommunications de Rennes (IETR), UMR CNRS 6164, \\ University of Rennes 1, Rennes, France \\ francesco.scattone@univ-rennes1.fr \\ ${ }^{2}$ Antenna and Sub-Millimetre Wave Section, ESTEC/ESA, Noordwijk Zh, The Netherlands
}

\begin{abstract}
This paper describes the design of a planar FabryPerot leaky wave antenna generating a flat-topped radiation pattern. The flat-topped pattern is achieved by exciting various leaky-wave modes between a metallic ground plane and a superstrate. Two configurations for the superstrate structure are proposed and analyzed: i) dielectric layers; ii) impedance sheets. In all cases, the antennas are fed by a small waveguide opening in the ground plane. An in-house optimization tool based on a Green's function spectral approach is used to derive the appropriate arrangement for the generation of the required flattopped pattern. The proposed approach is validated numerically for an antenna generating a flat topped pattern with a beamwidth of $\pm 25^{\circ}$. The proposed flat-topped antenna may be used for the design of phased arrays with reduced scan losses over a given angular range.
\end{abstract}

Index Terms-leaky-wave antennas, phased array antennas, flat-topped element pattern.

\section{INTRODUCTION}

Planar leaky-wave antennas (LWA) have been studied for decades as efficient and low cost antenna solutions [1]. During the years, different configurations have been proposed for various applications in the millimeter wave range. Here we will focus our attention on the so-called Fabry-Perot leaky-wave antennas. In this case an arrangement of dielectric substrates or impedance surfaces is placed over a metallic ground plane in order to enhance and/or modify the radiation properties of simple elementary sources. In particular, the possibility to enhance the gain of simple sources in the broadside direction has been widely investigated in literature [2]-[10]. Pattern shaping using leaky-wave modes and Fabry-Perot antennas have been also proposed in [11]-[13] for maximizing the aperture efficiency and edge of coverage gain of reflector systems.

In this paper an approach similar to that proposed in [11] will be used to generate flat-topped radiation patterns with Fabry-Perot LWA antennas. Flat-topped radiation patterns are suited as element pattern for phased arrays designed to scan their beams over a given field of view. In particular, the flattopped element pattern allows to reduce scan losses resulting

F. Scattone, M. Ettorre and R. Sauleau would like to thank Brittany Region (ARED projet 2012) and ESA (Co-sponsored PhD Ref. 267-2012) for supporting the PhD activity. The authors also acknowledge GENCI-IDRIS (grant 2013-05779).

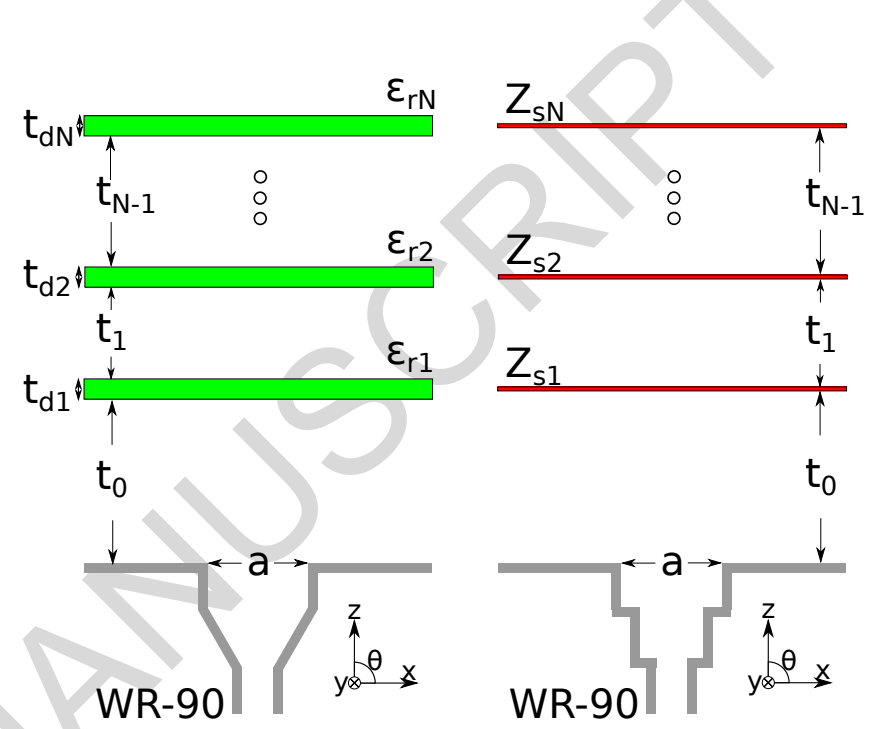

(a)

(b)

Fig. 1. Side view of the considered Fabry-Perot leaky-wave antennas. (a) Dielectric substrates. (b) Impedance surfaces.

from the appearance of grating lobes in the visible range [14], [15]. The structures considered here are shown in Fig. 1. In all cases, the antenna is fed by a square waveguide opening in the metallic ground plane. As a difference with [11] and [15] an optimization tool is used to derive directly the antenna configuration fulfilling the pattern requirements.

The paper is organized as follows. Sec. II introduces the optimization tool used for the shaping of the radiation pattern. Sec. III shows two designs of Fabry-Perot LWAs with a flattopped radiation pattern in the angular range $\theta= \pm 25^{\circ}$ using two kinds of superstrates: 1) dielectric substrates; 2) inductive impedance sheets. In all cases, the operating frequency is equal to $f_{0}=10 \mathrm{GHz}$. The proposed designs are validated by fullwave simulations. Finally conclusions are drawn in Sec. IV.

\section{OPTIMIZATION TOOL}

An optimization tool has been developed for shaping the radiation pattern of planar leaky-wave antennas. The aim of this tool is to find the antenna based on a superstrate configuration as in Fig. 1. 
The total electric field radiated by the two structures is considered. In the following we will focus our attention on the possibility to shape the total radiated field in the Eand H-planes. However, the same approach may be used to control the radiated field over other planes or for all azimuthal angles. The critical point of the optimization procedure is a fast evaluation of the radiation pattern of the structures under analysis. A Green's function spectral approach is adopted here [11] speeding up the computational time of the optimization tool. The electric field radiated by a generic magnetic current distribution over the ground plane of the structure can be expressed as:

$$
\mathbf{E}(x, y, z)=\frac{1}{|V|} \frac{1}{(2 \pi)^{2}} \int_{-\infty}^{\infty} \int_{-\infty}^{\infty} \underline{\underline{\mathbf{G}}}^{e m}\left(V_{T E}, V_{T M}, k_{x}, k_{y}, z\right) .
$$

$$
\mathbf{M}\left(k_{x}, k_{y}\right) e^{-j\left(k_{x} x+k_{y} y\right)} d k_{x} d k_{y}
$$

with $k_{x}$ and $k_{y}$ the spectral variables. $V=\sqrt{(a b) / 2}$ is the reference voltage and it depends on the lateral dimensions $a$ and $b$ of the feeding waveguide. $\mathbf{M}$ is the Fourier transform of the equivalent magnetic current distribution generated by the feeding systems. In the follwing we will consider a double slots configuration [11] and a waveguide opening in the ground plane. $\underline{\mathbf{G}}^{e m}$ is the spectral dyadic Green's function associated to the electric field for magnetic currents where, $V_{T E / T M}$ are the voltages of the pertinent transmission line representation of the structure under analysis. Transverse electric $(T E)$ and magnetic $(T M)$ modes are considered. Note that $V_{T E / T M}$ are function of the spectral variables and vertical position in $z$. The dyadic Green's is given by [11]:

$$
\begin{aligned}
& \underline{\underline{\mathbf{G}}}^{e m}\left(k_{x}, k_{y}, z\right)= \\
& {\left[\begin{array}{cc}
\frac{k_{x} k_{y}\left(V_{T M}\left(k_{\rho}\right)-V_{T E}\left(k_{\rho}\right)\right)}{k_{\rho}^{2}} & -\frac{\left(k_{x}^{2} V_{T M}\left(k_{\rho}\right)+k_{y}^{2} V_{T E}\left(k_{\rho}\right)\right.}{k_{\rho}^{2}} \\
\frac{k_{x}^{2} V_{T E}\left(k_{\rho}\right)+k_{y}^{2} V_{T M}\left(k_{\rho}\right)}{k_{\rho}^{2}} & \frac{k_{x} k_{y}\left(V_{T E}\left(k_{\rho}\right)-V_{T M}\left(k_{\rho}\right)\right)}{k_{\rho}^{2}} \\
-\frac{k_{y} V_{T M}\left(k_{\rho}\right)}{k_{z 0}} & \frac{k_{x} V_{T M}\left(k_{\rho}\right)}{k_{z 0}}
\end{array}\right]}
\end{aligned}
$$

Since we are interested in the far field of the considered structure, the radiated electric field is evaluated in the asymptotic form as:

$$
\begin{aligned}
\mathbf{E}(r, \theta, \phi) & \approx j k_{0} \cos \theta \frac{e^{-j k_{0} r}}{|V| 2 \pi r} . \\
& \underline{\underline{\mathbf{G}}}^{e m}\left(V_{T E}, V_{T M}, k_{x 0}, k_{y 0}, z_{u}\right) \mathbf{M}\left(k_{x 0}, k_{y 0}\right)
\end{aligned}
$$

with $k_{0}$ the free space wavelength, $k_{x 0}=k_{0} \sin \theta \cos \phi, k_{y 0}=$ $k_{0} \sin \theta \sin \phi$ and $z_{u}=\sum_{i=1}^{N} t_{i-1}+t_{d i}$ and $z_{u}=\sum_{i=1}^{N} t_{i-1}$ for the configuration in Fig. 1 (a) and Fig. 1 (b), respectively. The classical spherical coordinate reference system $(r, \theta, \phi)$ has been considered as shown in Fig. 1. Eq. 3 is used all along the optimization procedure.

The first step of the optimization procedure is the definition of the required flat-topped radiation pattern in the two principal planes. The requirements on the radiation pattern define a mask for the total electric field $\mathbf{E}(r, \theta, \phi)$, in the two principal planes as:

$$
\begin{aligned}
& \operatorname{Mask}\left(\theta, \phi_{0}\right)= \\
& \left\{\begin{array}{l}
\frac{\left|\mathbf{E}\left(r, \theta, \phi_{0}\right)\right|}{\max \left(\left|\mathbf{E}\left(r, \theta, \phi_{0}\right)\right|\right)}>C_{c} \quad \text { in } 0 \leq|\theta| \leq \theta_{c} \text { and } \phi_{0}=0,90^{\circ} \\
\frac{\left|\mathbf{E}\left(r, \theta, \phi_{0}\right)\right|}{\max \left(\left|\mathbf{E}\left(r, \theta, \phi_{0}\right)\right|\right)}<C_{s} \quad \text { in } \theta_{s} \leq|\theta| \leq 90^{\circ} \text { and } \phi_{0}=0,90^{\circ}
\end{array}\right.
\end{aligned}
$$

where $0<C_{s}<C_{c}<1$ are fixed limits for the normalized module of the total electric field, and $0<\theta_{c}<\theta_{s}<90^{\circ}$ are angular limits for the flat-topped radiation pattern in the E- and H-planes. In particular $\theta_{c}$ and $C_{c}$ controls the main beam of the flat-topped radiation pattern whereas, $\theta_{s}$ and $C_{s}$ its side lobe characteristics. The function $\max ()$ indicates the maximum value of the quantity within brackets. Note that the E- and H-plane corresponds to the $y z$-plane $\left(\phi=90^{\circ}\right)$ and $x z$ plane $(\phi=0)$, respectively. The provided mask also defines a reference solution Ref in the two principal planes equal to $C_{c}$ and $C_{s}$ for $0<|\theta|<\theta_{c}$ and $\theta_{s}<|\theta|<90^{\circ}$, respectively.

The range and step of variation of the design parameters of the considered structure are then provided. For the LWA made by dielectric layers (Fig. 1 (a)), the design parameters are: thicknesses of the dielectric substrate $\left(t_{d n}\right)$, dielectric permittivity $\left(\epsilon_{r}\right)$, number of layers $(N)$ of the stratification; whereas for the LWA made by impendence sheets (Fig. 1 (b)), the design parameters are: impedance surface $\left(Z_{s n}\right)$, periodicity $\left(t_{n}\right)$, number of layers $(N)$ of the stratification. A double slot configuration has been used as feeding element for the the superstate configuration made by dielectrics as in [11]. On the other hand, a simple waveguide aperture of size $a \times b$ has been used for the impedance sheet case. The feed parameters have been also included in the optimization procedure. An exhaustive search is made within the defined sets of variation for the design parameters for deriving the far field pattern fulfilling the required mask. Such a direct approach is possible thanks to fast evaluation of the radiated far field. However, other optimization schemes may be adopted to further speed up the optimization process. For each combination $(q)$ of the design parameters and two reference planes, the squared error of the derived far field pattern is derived as:

$$
\Delta^{(q)}=\int_{\theta_{\text {err }}}\left|\frac{\left|\mathbf{E}^{(q)}(\mathbf{r})\right|}{\max \left(\left|\mathbf{E}^{(q)}(\mathbf{r})\right|\right)}-\operatorname{Ref}\right|^{2},
$$

with $\theta_{\text {err }}$ the angular region where the derived field does not fulfill the required mask. A solution is found when the derived far-field pattern lays within the imposed mask or the squared error $\Delta^{(q)}$ is lower than a certain threshold. 


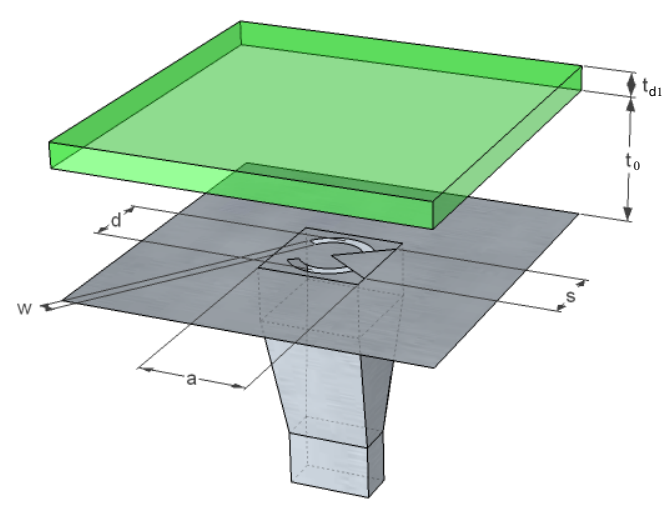

Fig. 2. Dielectric Fabry-Perot antennas: 3D view of the structure.

\section{FLAT-TOPPED RADIATION PATTERN}

The optimization procedure proposed in Sec. II is used here to design two LWA structures generating a flat-topped radiation patten and made by two kinds of superstrates: 1) dielectric substrates; 2) inductive impedance sheets. The operating frequency is chosen equal to $f_{0}=10 \mathrm{GHz}$. The following mask is imposed on the radiation pattern in two principal planes:

$$
\begin{aligned}
& \operatorname{Mask}\left(\theta, \phi_{0}\right)= \\
& \left\{\begin{array}{l}
\frac{\left|\mathbf{E}\left(r, \theta, \phi_{0}\right)\right|}{\max \left(\left|\mathbf{E}\left(r, \theta, \phi_{0}\right)\right|\right)}>0.71 \quad \text { in } 0 \leq|\theta| \leq 25^{\circ} \text { and } \phi_{0}=0,90^{\circ} \\
\frac{\left|\mathbf{E}\left(r, \theta, \phi_{0}\right)\right|}{\max \left(\left|\mathbf{E}\left(r, \theta, \phi_{0}\right)\right|\right)}<0.18 \quad \text { in } 50^{\circ} \leq|\theta| \leq 90^{\circ} \text { and } \phi_{0}=0,90^{\circ}
\end{array}\right.
\end{aligned}
$$

Note that this mask is chosen as an example. However, other requirements may be defined based on the considered target application [14], [15].

\section{A. Dielectric superstrate}

The geometry of the dielectric based Fabry-Perot LWA is shown in Fig. 1 (a). The range of variation for the various design parameters has been chosen equal to: $\lambda / 4 \leq t_{i}, t_{d i} \leq$ $5 / 4 \lambda, 16 \mathrm{~mm} \leq a, b \leq 30 \mathrm{~mm}, 2 \leq \epsilon_{r} \leq 12$. The maximum number of dielectric layer $N$ was set to 3 .

The antenna structure resulting from the optimization process is shown in Fig. 2. It is made by one dielectric substrate of permittivity $\epsilon_{r}=5$. The dielectric substrate thicknesses is $t_{d 1}=11.7 \mathrm{~mm}$ and is placed at $t_{0}=14.6 \mathrm{~mm}$ from the ground plane. It is worth noting that $t_{0}$ does not assume an integer multiple value of $\lambda_{0} / 2$ (free-space wavelength, $\lambda_{0}=30 \mathrm{~mm}$ ) as in [2], leading to good results both in bandwidth and in reflection coefficient (input reflection coefficient lower than $-10 \mathrm{~dB}$ in a $16 \%$ bandwidth around the central frequency $f_{0}$ ) despite of the high permittivity of the substrate. The final size of the double slot configuration are $d=18 \mathrm{~mm}, s=25 \mathrm{~mm}$ and $w=2.5 \mathrm{~mm}$, (refer to Fig. 2). This feeding system has

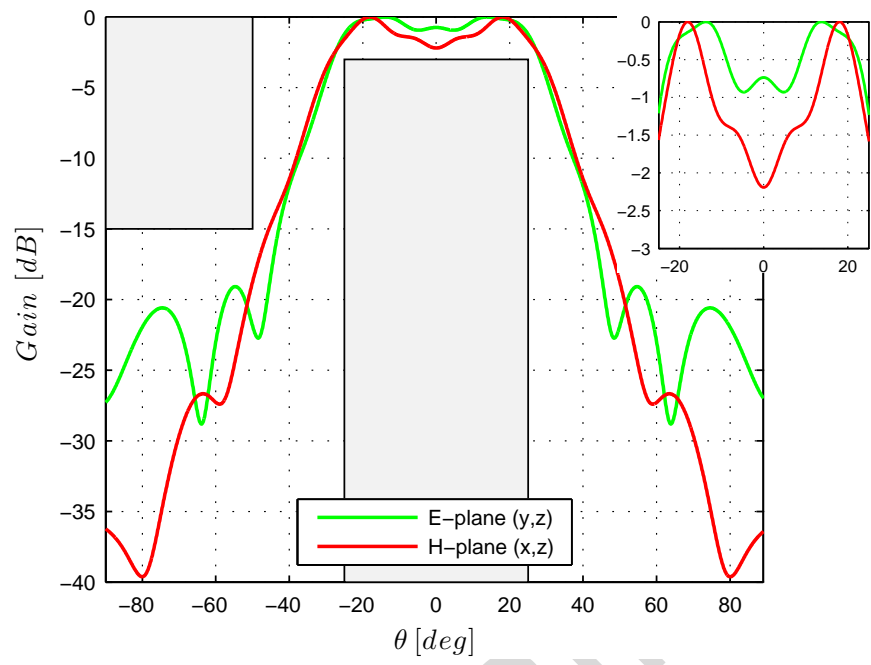

Fig. 3. Dielectric Fabry-Perot antennas: radiation patterns in the E- and $\mathrm{H}$-planes with a zoom of the central region.

been used to reduce the side lobe level of the final radiation pattern in the E-plane [11]. The two slots are fed in phase by a square waveguide with side length of $a=23 \mathrm{~mm}$. For feeding purposes, the waveguide is connected to a standard WR-90 waveguide by a tapered transition. Fig. 3 shows the radiation patterns in the E-plane $\left(\phi=90^{\circ}\right)$ and H-plane $\left(\phi=0^{\circ}\right)$ provided by HFSS. The radiation patterns are within the imposed mask. The mask is reported in Fig. 3 for clearness.

The dispersion diagrams of the obtained LWA structure are shown in Fig. 4 for a better understanding of its physical operation. It follows that the main couple of leaky-wave modes $\left(T M_{1}, T E_{1}\right)$ is not pointing at broadside at $f_{0}=10 \mathrm{GHz}$, but at $\theta=25^{\circ}$, i.e. at the angular limit $\theta_{c}$ of the flat-topped radiation pattern. The $T M_{0}$ mode generates a side lobe in the E-plane around $\theta=60^{\circ}$. This side lobe is suppressed

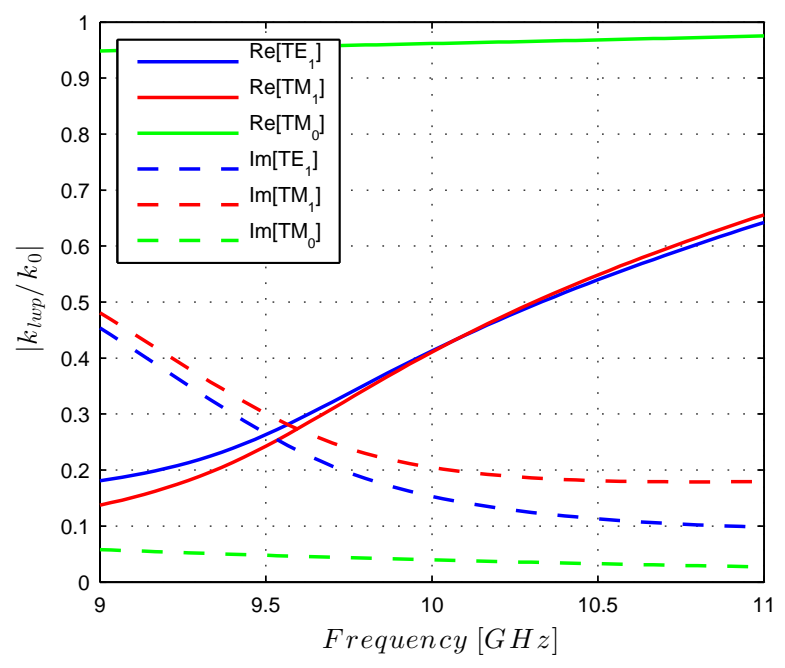

Fig. 4. Dielectric Fabry-Perot antennas: dispersion diagram. 


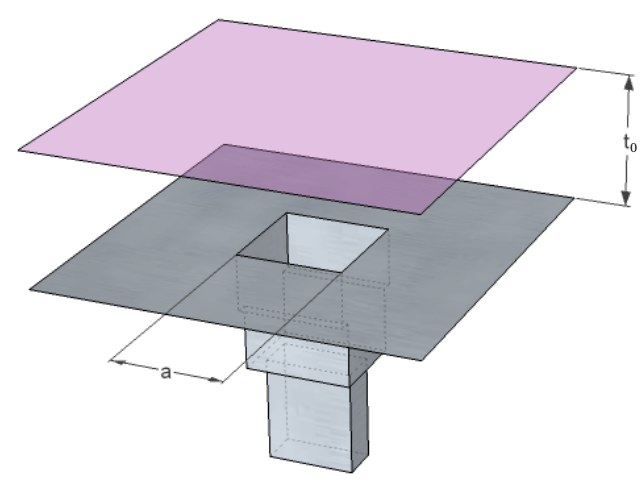

Fig. 5. Fabry-Perot antenna based on impedance sheets: 3D view of the structure.

using the first zero of the array factor of the double slot feed configuration.

\section{B. Impedance sheet}

The geometry of the sheet impedance based Fabry-Perot LWA is shown in Fig. 1 (b). The range of variation for the various design parameters has been chosen equal to: $\lambda / 4 \leq$ $t_{i} \leq 5 / 4 \lambda, 16 \mathrm{~mm} \leq a, b \leq 30 \mathrm{~mm}, j 10 \leq Z_{s i} \leq j 500 \mathrm{Ohm}$. The maximum number of dielectric layers $N$ was set equal to 3. Note that for brevity, only inductive surfaces have been considered. However, the procedure may be applied to capacitive surfaces as well.

The final results of the optimization procedure is a LWA made by a single impedance surface of $Z_{s}=j 220 \Omega$ at a distance $t_{0}=14.1 \mathrm{~mm}$ from the ground plane (Fig. 5). The feeding waveguide is made by a square waveguide with side length equal to $a=28 \mathrm{~mm}$. The latter is connected to a WR90 waveguide by a matching transition, as shown in Fig. 5.

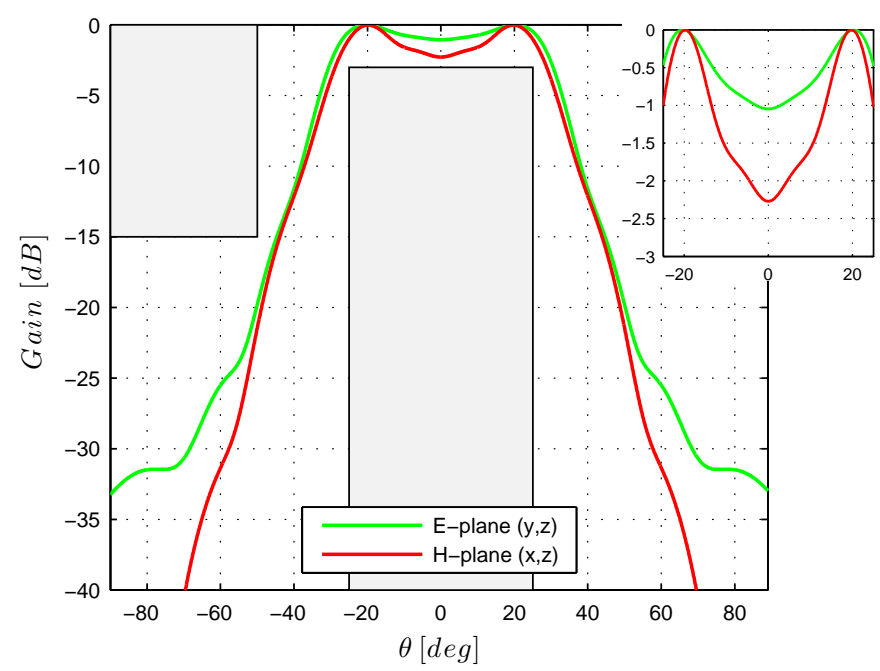

Fig. 6. Fabry-Perot antenna based on impedance sheets: radiation patterns in the E- and H-planes with a zoom of the central region.



Fig. 7. Fabry-Perot antenna based on impedance sheets: dispersion diagram.

The reflection coefficient is lower than $-10 \mathrm{~dB}$ in a $9 \%$ bandwidth around the operating frequency $f_{0}$. The radiation patterns obtained with HFSS are shown in Fig. 6. The patterns in the E-plane $\left(\phi=90^{\circ}\right)$ and H-plane $\left(\phi=0^{\circ}\right)$ are within the imposed mask. It is worth saying that the impedance surface can be implemented by using a periodic arrangement of slots over a metallic layer [5].

The dispersion diagram for the resulting structure is shown in Fig. 7. Also in this case, the main couple of leakywave modes $\left(T M_{1}, T E_{1}\right)$ is not pointing at broadside at the operating frequency $f_{0}$. As a difference with the previous case, the $T M_{0}$ is radiating toward endfire [15] and does not introduce a side lobe within the visible range.

\section{CONCLUSION}

An optimization tool for Fabry-Perot LWA generating flattopped radiation patterns have been proposed. The considered LWA structures are based on superstrate configurations of dielectric substrates and impedance sheets. The solution of the problem is obtained through an exhaustive search within a defined range of variation for the design parameters of the structures. The optimization tool uses a spectral Green's function approach to efficiently calculate the far field radiated by the LWA structures at each step of the optimization procedure speeding up the computational time. The final results of the optimization tool are the geometrical parameters of the considered configuration generating the required flattopped radiation pattern with defined radiation characteristics in terms of main beam and side lobe level. Two antennas have been designed to validate the proposed approach and validated by full-wave simulations. The two structures are made by a superstrate configuration of dielectric substrates and impedance surfaces, respectively. The resulting radiation patterns present a flat-topped behaviour in the angular range $0 \leq|\theta| \leq 25^{\circ}$ in E- and H-planes together with a low side 
lobe level $(<-15 \mathrm{~dB})$ and an important roll-off. The obtained LWA structures may be used as radiating element for phased arrays with periodicity higher than a wavelength for reducing the scan losses due to the appearance of grating lobes in the field of view.

\section{ACKNOWLEDGMENT}

The authors would like to thank Dr. Cyril Mangenot, from the European Space Agency, for valuable discussions related to this topic.

\section{REFERENCES}

[1] C. A. Balanis, "Modern antenna handbook," Wiley-Interscience New York, NY, USA, 2008.

[2] D. R. Jackson and A. A. Oliner, "A leaky-wave analysis of the highgain printed antenna configuration," IEEE Transactions on Antennas and Propagation, vol. 36, pp. 905-910, Jul. 1988.

[3] D. R. Jackson, A. A. Oliner, and A. Ip, "Leaky-wave propagation and radiation for a narrow-beam multiple-layer dielectric structure," IEEE Transactions on Antennas and Propagation, vol. 41, pp. 344-348, Mar. 1993.

[4] T. Zhao, D. R. Jackson, J. T. Williams, H. D. Yang, and A. A Oliner, "2-D periodic leaky-wave antennas- part I: metal patch Design," IEEE Transactions on Antennas and Propagation, vol. 53, no.11, pp. 35053514, Nov. 2005.

[5] T. Zhao, D. R. Jackson, and J. T. Williams, "2-D periodic leaky-wave antennas part II: slot design," IEEE Transactions on Antennas and Propagation, vol. 41, pp. 3515-3524, Nov. 2005.

[6] R. Sauleau, Ph. Coquet, D. Thouroude, J.-P. Daniel, and T. Matsui, "Radiation characteristics and performance of millimeter wave horn-fed gaussian beam antennas," IEEE Transactions on Antennas and Propagation, vol. 51., pp. 378-387, Mar. 2003

[7] R. Sauleau, Ph. Coquet, T. Matsui, and J.-P. Daniel, "A new concep of focusing antennas using plane-parallel Fabry-Perot cavities with nonuniform mirrors," IEEE Transactions on Antennas and Propagation, vol. 51, pp. 3171-3175, Nov. 2003

[8] G. Lovat, P. Burghignoli and D.R. Jackson, "Fundamental properties and optimization of broadside radiation from uniform leaky-wave antennas,' IEEE Transactions on Antennas and Propagation, vol. 54, pp. 1442-1452, May 2006.

[9] S.A. Hosseini, F. Capolino, F. De Flaviis, P. Burghignoli, G. Lovat and D.R. Jackson, "Improved method to estimate the $3 \mathrm{~dB}$ power bandwidth of a Fabry-Prot Cavity antenna covered by a thin frequency selective surface," IEEE International Symposium on Antennas and Propagation (APSURSI) 2011, pp. 1281,1284, 3-8 July 2011.

[10] P. Burghignoli, G. Lovat and D.R. Jackson, "Analysis and optimization of leaky-wave radiation at broadside from a class of 1-D periodic structures," IEEE Transactions on Antennas and Propagation, vol. 54, pp. 1442-1452, May 2006.

[11] A. Neto, N. Llombart, G. Gerini, D. M. Bonnedal, and P. De Maagt, "EBG enhanced feeds for the improvement of the aperture efficiency of reflector antennas," IEEE Transactions on Antennas and Propagation, vol. 55, pp. 2185-2193, Aug. 2007.

[12] N. Llombart, A. Neto, G. Gerini, D. M. Bonnedal, and P. De Maagt, "Leaky wave enhanced feed arrays for the improvement of the edge of coverage gain in multibeam reflector antennas," IEEE Transactions on Antennas and Propagation, vol. 56, pp. 1280-1291, May 2008.

[13] A. Neto, M. Ettorre, G. Gerini, and P. De Maagt, "Leaky wave enhanced feeds for multibeam reflectors to be used for Telecom satellite based links," IEEE Transactions on Antennas and Propagation, vol. 54, pp. 2593-2604, Sep. 2006.

[14] R. J. Mailloux, "An overlapped subarray for limited scan application," IEEE Transactions on Antennas and Propagation, vol. 22, pp. 487-489, May 1974

[15] D. Blanco, N. Llombart, E. Rajo-Iglesias, "On the use of leaky wave phased arrays for the reduction of the grating lobe level," forthcoming on IEEE Transactions on Antennas and Propagation. 